\title{
Optimizing the controllability of arbitrary networks with genetic algorithm
}

\author{
Xin-Feng Li, Zhe-Ming Lu* \\ School of Aeronautics and Astronautics, Zhejiang University, Hangzhou,310027, China
}

\begin{abstract}
Recently, as the controllability of complex networks attracts much attention, how to optimize networks' controllability has become a common and urgent problem. In this paper, we develop an efficient genetic algorithm oriented optimization tool to optimize the controllability of arbitrary networks consisting of both state nodes and control nodes under Popov-Belevitch-Hautus rank condition. The experimental results on a number of benchmark networks show the effectiveness of this method and the evolution of network topology is captured. Furthermore, we explore how network structure affects its controllability and find that the sparser a network is, the more control nodes are needed to control it and the larger differences between node degrees, the more control nodes are needed to achieve the full control. Our framework provides an alternative to controllability optimization and can be applied to arbitrary networks without any limitations.
\end{abstract}

Keywords: complex networks, controllability optimization, optimal topology, genetic algorithm PACS: 89.75. Fb, 89.75.Hc, 89.75.-k, 87.55.de

2000 MSC: 93B05, 90C10, 05C 82

\section{Introduction}

Complex networks are ubiquitous in nature and society [1-4], describing various systems such as food webs [5], biochemical networks [6, 7], social networks [8], citation networks [9, 10], and so on. Discovering their behind principles will enrich our understanding of complex natural and human systems. Previous research has mostly been focused on modeling [11, 12], measuring [4], synchronization [13], navigation and search [14], community detection [15-17], epidemic spreading [18-20], robustness [21, 22], and so on.

However, the ultimate proof of our understanding of complex systems is reflected in our ability to control them [23], which means injecting the external signals to some suitable nodes (called driver nodes) so that the system can be driven from any initial state to any desired final state within finite time. Considerable efforts [24-29] have been devoted to uncover the relationship between network topology and its controllability. The ground-breaking contribution was made by Liu et al. [23] who developed a minimum input theory to efficiently characterize the structural controllability of directed networks, allowing the minimum number of driver nodes

\footnotetext{
*Corresponding author: Zhe-Ming Lu. Tel: +86 13868074895

Email address: zheminglu@zju.edu. cn (Zhe-Ming Lu )

Preprint submitted to Physica A: Statistical Mechanics and its Applications
}

November 30, 2015 
$\left(N_{D}\right)$ to be identified to achieve full control. Their simulation results show that $N_{D}$ is mainly determined by networks' degree distribution and the sparse inhomogeneous networks are the most difficult to control. The structural controllability framework opens a great avalanche of research on network controllability, several basic issues have been carefully addressed, such as linear edge dynamics [30], lower and upper bounds of energy required for control [31], control centrality [32], robustness of controllability [33], and so on.

In addition to the above issues, how to optimize the controllability of networks has become a common and urgent problem. Wang et al. [34] first investigate this problem and propose a perturbation strategy based on adding the least number of edges (only $N_{D}-1$ edges) for connecting the separated control paths in a proper sequence, which brings the network under control with only one driver node. Despite of its excellent performance, adding edges disturbs the network topology and is not practical and economic in practice. Thus Hou et al. [35] propose an efficient heuristic approximation algorithm to assign edge direction based on node residual degree, which neither changes the original structure of network nor pays additional cost for adding links. However, this algorithm cannot guarantee the optimal orientation of controllability for the given network topology because of its random feature of selecting nodes or edges. To find the optimal edge orientation, Xiao et al. [36] systematically study the edge orientation of optimal controllability problem and transfer this problem to find the maximum independent set of the constructed switching network. Besides, Iudice et al. [37] present an exact method to optimize the network controllability based on the solution of an integer linear programming (ILP) in which some physical and economic constraints are considered. All the above studies assume that the link weights of networks are precisely unknown and the networks taken into consideration only consist of state nodes. However, most real-world complex systems physically consist of both state nodes and control nodes and the linking strength between nodes could be reasonably determined by principle knowledge. Therefore, Ding et al. [38] propose the problem of optimizing the controllability of networks consisting of both "state nodes" and "control node", and develop an extremal optimization oriented heuristic tool to find the optimal network topology with the minimum number of control nodes while maintaining the network's full controllability under Kalman rank condition [39].

Although Ding et al.'s work [38] offers a general tool to optimize the controllability of networks consisting of both state nodes and control nodes, their method is only valid for directed networks. In order to speed up the algorithm, the authors take structural controllability framework [23] as a pre-test. However, the structural controllability framework is only applicable to directed networks characterized by structural matrices in which all links are represented by independent free parameters [40]. For undirected networks or networks where exact link weights are available, the assumption of structural matrix is violated, thus this method fails.

To overcome this limitation, in this paper we develop an efficient genetic algorithm oriented heuristic tool to optimize the controllability of networks consisting of both state nodes and control nodes with arbitrary structures and link weights on the basis of Popov-Belevitch-Hautus (PBH) rank condition [41], which is equivalent to Kalman rank condition [39]. The experimental results on a number of benchmark networks show the effectiveness of this method and the evolution process of network topology is captured. Furthermore, we investigate how network's structural properties affect its controllability.

The rest of the paper is organized as follows. In Section 2, we introduce the preliminary results of network controllability. In Section 3, we give the problem formulation. In Section 4, we detail the genetic algorithm oriented optimization framework. In section 5, simulation results and discussions are presented. Finally, Section 6 concludes the paper. 


\section{Preliminaries}

Consider a network of $N$ nodes described by the following ordinary differential equation [23]:

$$
\dot{\mathbf{x}}=A \mathbf{x}+B \mathbf{u}
$$

where $\mathbf{x}=\left(x_{1}, x_{2}, \ldots, x_{N}\right)^{T}$ captures the states of $N$ nodes, $A \in \mathbb{R}^{N \times N}$ denotes the coupling matrix of the system, in which $a_{i j}$ represents the weight of directed link from node $x_{j}$ to $x_{i}$ (for undirected networks, $\left.a_{i j}=a_{j i}\right) . \mathbf{u}=\left(u_{1}, u_{2}, \ldots, u_{P}\right)^{T}$ is the input or control vector of $P$ controllers, and $B \in \mathbb{R}^{N \times P}$ is the input matrix, in which $b_{i j}$ represents the weight of directed link from control node $u_{j}$ to state node $x_{i}$.

The system described by Eq.(1) is said controllable if it can be driven from any initial state to any desired final state within finite time. According to the classic Kalman rank condition [39], that is possible if and only if the $N \times N P$ controllability matrix

$$
C=\left(B, A B, A^{2} B, \ldots, A^{N-1} B\right)
$$

has full rank, that is

$$
\operatorname{rank}(C)=N
$$

The standard approach to the controllability problem is to find a suitable matrix $B$ consisting of the minimum number of columns so as to satisfy the Kalman rank condition. However, a practical difficulty is that there are $2^{N}$ possible combinations of placing controllers and the weight of links are often not known precisely. Therefore, the concept of structural controllability [42] is introduced to overcome this difficulty [23].

Eq.(1) is structurally controllable if it is possible to assign values to the nonzero entries of the matrices $A$ and $B$ such that (3) is verified. Note that a structurally controllable system is controllable for almost all weight combinations, except for some pathological cases with zero measure that occur when the system parameters satisfy certain accidental constraints [42, 43]. By introducing the structural controllability to complex networks, Liu et al. [23] proposed the structural controllability framework and proved that the minimum number of driver nodes needed to maintain network's structural controllability is determined by its maximum matching where external control is necessary for every unmatched node. As the maximum matching [44] in directed networks can be efficiently identified in $O(\sqrt{N} L)$ time, where $L$ is the number of links between state nodes, Liu et al.'s framework offers an efficient method to determine the minimum number of driver nodes for an arbitrary directed network [23]. However, the drawback is that the structural controllability framework is only applicable to directed networks characterized by structural matrices, in which all links are represented by independent free parameters [40]. This requirement may be violated if exact link weights are given or by the symmetric characteristic of undirected networks, resulting in the failure of this approach.

An alternative criterion that is equivalent to the Kalman rank condition is called PBH rank condition [45-48], which stipulates that the system (Eq.(1)) is controllable if and only if

$$
\operatorname{rank}\left(c I_{N}-A, B\right)=N
$$

is satisfied for any complex number $c$, where $I_{N}$ is the identity matrix of dimension $N$. It can be further proved that, if and only if any eigenvalue $\lambda$ belonging to $A$ satisfies Eq.(4), full control can be ensured [40], i.e.

$$
\operatorname{rank}\left(\lambda_{i} I_{N}-A, B\right)=N, \quad \forall \lambda_{i} \in \operatorname{eig}(A)
$$


where $\operatorname{eig}(A)$ is the set of all the distinct eigenvalues of $A$. In terms of the rank inequality, Eq.(5) can be rewritten as

$$
N=\operatorname{rank}\left(\lambda_{i} I_{N}-A, B\right) \leq \operatorname{rank}\left(\lambda_{i} I_{N}-A\right)+\operatorname{rank}(B)
$$

such that

$$
\operatorname{rank}(B) \geq N-\operatorname{rank}\left(\lambda_{i} I_{N}-A\right), \quad \forall \lambda_{i} \in \operatorname{eig}(A)
$$

Eq.(7) indicates that if the system (Eq.(1)) is controllable then $\operatorname{rank}(B)$ must be larger than or equal to the maximum value of $N-\operatorname{rank}\left(\lambda_{i} I_{N}-A\right)$ for all eigenvalues $\lambda_{i}$, in other words, if a control matrix $B$ does not satisfy Eq.(7) the system will never be controllable. Therefore, we can directly exclude all the control matrices against Eq.(7) without the detailed verification of PBH rank condition. As the maximum value of $N-\operatorname{rank}\left(\lambda_{i} I_{N}-A\right)$ is fixed for a given network, this exclusion can be done efficiently by simply calculating $\operatorname{rank}(B)$. In contrast, when using the Kalman rank condition, one has to reconstruct the large $N \times N P$ controllability matrix with massive matrix multiplications for every candidate control matrix $B$ without any shortcut. In addition, the rank computation of PBH matrix $(N \times(N+P))$ is much faster than that of Kalman matrix $(N \times N P)$.

We conclude this section with the comparisons of different controllability approaches. The structural controllability framework can efficiently determine the minimal driver nodes based on the maximum matching algorithm. However, it is not applicable to undirected networks or networks if exact link weights are given. Both the Kalman rank condition and PBH rank condition can be applied to arbitrary networks without any limitations. Nevertheless, the latter can efficiently exclude many uncontrollable configurations without detailed verifications whereas the former cannot. Taking into account the wide applicable scope as well as the efficient exclusion strategy, we choose the $\mathrm{PBH}$ rank condition as criterion in this paper.

\section{Problem formulation}

In this section, we give the formal description of controllability optimization problem over networks consisting of both state nodes and control nodes.

Definition 3.1. A network consisting of both state nodes and control nodes can be represented by a triple tuple $G=(V, E, W)$ with node set $V=V_{A} \cup V_{B}$ and edge set $E=E_{A} \cup E_{B}$. Here, $V_{A}=\left\{v_{1}, v_{2}, \ldots, v_{N}\right\}=\left\{x_{1}, x_{2}, \ldots, x_{N}\right\}$ is the set of state nodes, $V_{B}=\left\{v_{N+1}, v_{N+2}, \ldots, v_{N+P}\right\}=$ $\left\{u_{1}, u_{2}, \ldots, u_{P}\right\}$ is the set of control nodes. $E_{A} \subseteq V_{A} \times V_{A}$ is the set of edges between state nodes, $E_{B} \subseteq V_{B} \times V_{A}$ is the set of edges between control nodes and state nodes. $W \in \mathbb{R}^{(N+P) \times(N+P)}$ is the matrix of link weights, $w_{i j}=0$ if $v_{j}$ does not have a link to $v_{i}$, i.e., node- $j$ does not affect node- $i$. Otherwise, $w_{i j}$ gives the strength or weight that node- $j$ can affect node- $i$. Positive (or negative) value of $w_{i j}$ means the link ( $\left.j \rightarrow i\right)$ is excitatory (or inhibitory) (see Fig. 1 as an example).

Remark 3.1. The dynamics taking place on $G(V, E, W)$ can be described by Eq.(1) where $A \in$ $\mathbb{R}^{N \times N}, B \in \mathbb{R}^{N \times P}$ are sub-blocks of $W$, i.e., $W=\left[\begin{array}{cc}A & B \\ 0 & 0\end{array}\right]$.

Definition 3.2. A control scheme of $G(V, E, W)$ is a combination of control nodes, denoted by a binary diagonal matrix $D=\operatorname{diag}\left(d_{1}, d_{2}, \ldots, d_{P}\right)$ where $d_{j}(j=1,2, \ldots P)$ is a $0-1$ variable, indicating the status of corresponding control node $u_{j}, d_{j}=1$ means $u_{j}$ is selected to form the desired network topology, otherwise removed from the network along with the associated connections. 


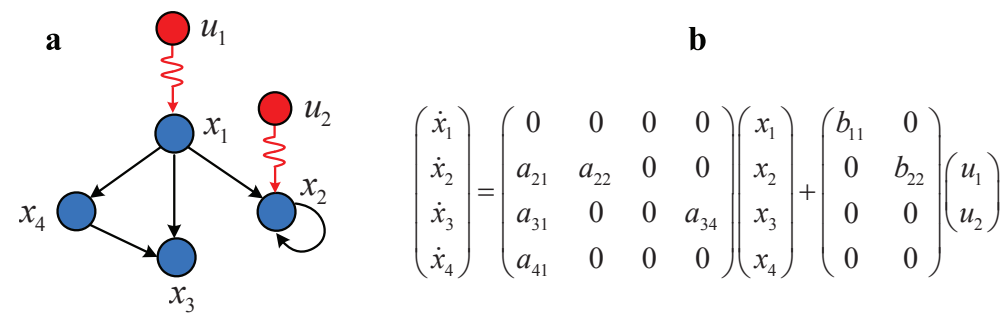

Figure 1: (a) A simple directed network and (b) its corresponding dynamics, where $V_{A}=\left\{x_{1}, x_{2}, x_{3}, x_{4}\right\}, V_{B}=\left\{u_{1}, u_{2}\right\}$, $E_{A}=\left\{\left(x_{1}, x_{2}\right),\left(x_{1}, x_{3}\right),\left(x_{1}, x_{4}\right),\left(x_{2}, x_{2}\right),\left(x_{4}, x_{3}\right)\right\}, E_{B}=\left\{\left(u_{1}, x_{1}\right),\left(u_{2}, x_{2}\right)\right\}$.

Remark 3.2. It is clear to see that a specific control scheme $D^{*}$ corresponds to a set of selected control nodes $U^{*}=\left\{u_{j} \mid d_{j}=1, j=1,2, \ldots, P\right\}$ and determines a network topology $G^{*}\left(V^{*}, E^{*}, W^{*}\right)$, where $V^{*} \subseteq V, E^{*} \subseteq E, W^{*} \in \mathbb{R}^{(N+r) \times(N+r)}$, in which $r$ is the number of selected control nodes. The dynamics taking place on $G^{*}\left(V^{*}, E^{*}, W^{*}\right)$ is therefore changed to

$$
\dot{\mathbf{x}}=A \mathbf{x}+B^{*} \mathbf{u}^{*}
$$

where $B^{*} \in \mathbb{R}^{N \times r}$ represents the new input matrix between $r$ selected control nodes and $N$ state nodes, and $\mathbf{u}^{*} \in \mathbb{R}^{r}$ denotes the time-dependent control signals of $r$ selected control nodes.

Definition 3.3. Indicator matrix $M \in \mathbb{R}^{P \times r}$ depicts the index of selected control nodes, where $m_{i j}=1$ denotes the $j$ th selected control node is $u_{i}, i=1,2, \ldots, P, j=1,2, \ldots, r$.

Remark 3.3. $M$ is comprised of all the non-zero columns in $D^{*}$. For example, suppose we choose $U^{*}=\left\{u_{1}, u_{2}, u_{4}\right\}$ from $U=\left\{u_{1}, u_{2}, u_{3}, u_{4}\right\}$, then the corresponding $M$ is as follows,

$$
M=\left[\begin{array}{lll}
1 & 0 & 0 \\
0 & 1 & 0 \\
0 & 0 & 0 \\
0 & 0 & 1
\end{array}\right]
$$

Remark 3.4. The new input matrix $B^{*}$ and the new input vector $\mathbf{u}^{*}$ can be rewritten as $B^{*}=$ $B D^{*} M$ and $\mathbf{u}^{*}=M^{T} \mathbf{u}$, respectively. By substituting $B^{*}$ and $\mathbf{u}^{*}$ into Eq.(8), we have

$$
\dot{\mathbf{x}}=A \mathbf{x}+B D^{*} M\left(M^{T} \mathbf{u}\right)
$$

Fig. 2 demonstrates how the control scheme $D^{*}$ can affect the formation of new network topology.

Remark 3.5. According to the PBH rank condition (Eq.(5)), the new system (Eq.(9)) is controllable, if and only if

$$
\operatorname{rank}\left(\lambda_{i} I_{N}-A, B D^{*} M\right)=N
$$

is satisfied for any eigenvalue $\lambda_{i}$ belonging to $A$.

Given an arbitrary network $G(V, E, W)$, our objective is to choose the minimal control nodes such that the formed network can be fully controlled. Therefore, we can formalize the problem of "control over an arbitrary network" as follows,

$$
\min _{D} \sum_{j=1}^{P} d_{j}
$$



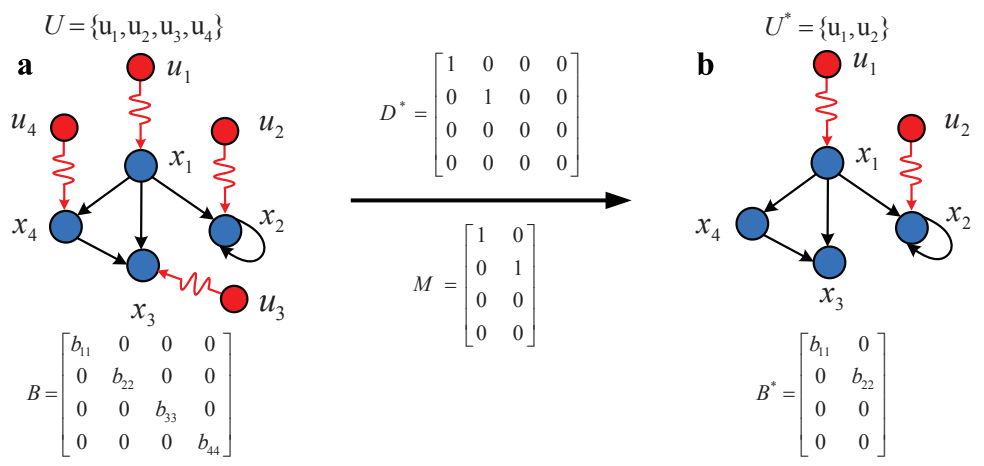

Figure 2: Impact of control matrix $D$ on forming new network topology. (a) The original network topology with 4 state nodes and 4 candidate control nodes. (b) The new network topology after choosing $\left\{u_{1}, u_{2}\right\}$ as control nodes, the corresponding new input matrix $B^{*}$ is obtained by $B^{*}=B D^{*} M$.

subject to

$$
\begin{gathered}
\operatorname{rank}\left(\lambda_{i} I_{N}-A, B D M\right)=N, \quad \forall \lambda_{i} \in \operatorname{eig}(A) \\
d_{j}=\{0,1\}, \quad j=1,2,3 \ldots, P
\end{gathered}
$$

Eq.(12) states the complete controllability constraint under PBH rank condition, where $A$ is the initial state matrix, $B$ is the initial input matrix, $M$ denotes the indicator matrix, $D$ represents a control scheme, $\lambda_{i}(i=1,2, \ldots, l)$ is the eigenvalue of $A$, eig $(A)$ is the set of distinct eigenvalues of $A, l$ is the cardinality of $\operatorname{eig}(A)$.

\section{Solution framework}

\subsection{Overview of genetic algorithm}

Genetic algorithm [49] (GA) is a heuristic search algorithm based on the evolutionary idea of natural selection and has been widely used to solve global optimization problems. In general, GA tends to work better than the traditional optimization algorithms because it does not make use of single-point transition rules to move from one single instance to another. Instead, it takes advantage of an entire set of solutions spread throughout the solution space, all of which are experimenting upon many potential optima.

In GA, a population of individuals is maintained within the search space, each representing a possible solution to a given problem. Each individual is coded as a finite length vector of variables (usually a binary 01 string) and has a fitness score to measure how good a solution it is to the particular problem. To continue the genetic analogy the individuals are likened to chromosomes and the variables are analogous to genes. Starting with a randomly generated population of chromosomes, GA carries out a process of fitness-based selection and recombination to evolve toward a better population. During recombination, parent chromosomes are selected to produce children chromosomes according to their fitness scores - the better the chromosomes are, the more chances they have to be selected. As a result, highly fit solutions are given more opportunities to reproduce so that offspring inherit characteristics from each parent, whereas poorly fit solutions die out and are replaced by the new solutions, eventually creating a new generation. Usually, a little random mutation helps to prevent the population from being filled up with numerous copies of the same solution. Once the population has converged and is not producing 
offspring noticeably different from those in previous generations, the algorithm is said to have converged to a set of solutions to the problem. The individual with optimal (or near optimal) fitness score is then the objective solution.

As the standard GA has a tendency to converge towards local optima, GA with adaptive parameters (adaptive genetic algorithm) has been proposed [50], which utilizes the population information to adjust the crossover and mutation probability adaptively so as to maintain the population diversity as well as to sustain the convergence capacity. In this way, high-fitness solutions are protected whereas low-fitness solutions are disrupted.

\subsection{Workflow of GA for the network controllability optimization problem}

In order to apply GA, an appropriate encoding scheme should be presented. As the candidate solution $D$ is a diagonal matrix with $d_{j} \in\{0,1\}$, the chromosome can be encoded as a binary string by extracting all the diagonal elements, as depicted in Fig.3. For example, suppose we have a network with $P=5$ candidate control nodes, then a control scheme $D=\operatorname{diag}(1,1,0,1,0)$ is encoded as 11010 , indicating that node $u_{1}, u_{2}, u_{4}$ is selected to form the desired topology.

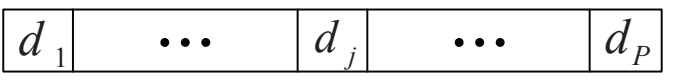

Figure 3: Structure of chromosome of the proposed GA.

From Eq.(11)-(13), we can see that the controllability optimization of complex networks is a typical 0-1 integer programming problem, our objective is to find a suitable control scheme $D$ such that Eq.(11) can achieve the minimum. Notice that there is a PBH controllability constraint (Eq.(12)) in this problem. For applying GA conveniently, we employ the penalty function method [51] to transform the constrained optimization into unconstrained optimization. To be specific, we define a penalty term $\operatorname{Pen}_{i}(D)$ to measure the violation of the $i$-th constraint for a specific $D$

$$
\operatorname{Pen}_{i}(D)=\sigma_{i} \times\left(\operatorname{rank}\left(\lambda_{i} I_{N}-A, B D M\right)-N\right)^{2}, \quad i=1,2,3 \ldots, l
$$

where $\sigma_{i}(=10 P)$ is the penalty coefficient, $l$ is the number of all the distinct eigenvalues of matrix $A$. The total penalty of $D$ is defined as $\operatorname{Pen}(D)=\sum_{i=1}^{l} \operatorname{Pen}_{i}(D)$. Note that if the control scheme $D$ makes the network fully controllable, $\operatorname{Pen}(D)$ will be zero, and vice versa. Then we incorporate the penalty item into the objective function (Eq.(11)) and define the cost function $f(D)$ as follows,

$$
\begin{aligned}
f(D) & =\sum_{j=1}^{P} d_{j}+\operatorname{Pen}(D) \\
& =\sum_{j=1}^{P} d_{j}+\sum_{i=1}^{l} \sigma_{i} \times\left(\operatorname{rank}\left(\lambda_{i} I_{N}-A, B D M\right)-N\right)^{2}
\end{aligned}
$$

It should be stressed that this is a minimization problem, the smaller the fitness, the better the solution. Fig.4 illustrates the fitness evaluation on a simple directed-weighted network.

Once the chromosome representation and the cost function are defined, the proposed GA for controllability optimization of complex networks proceeds as follows: 

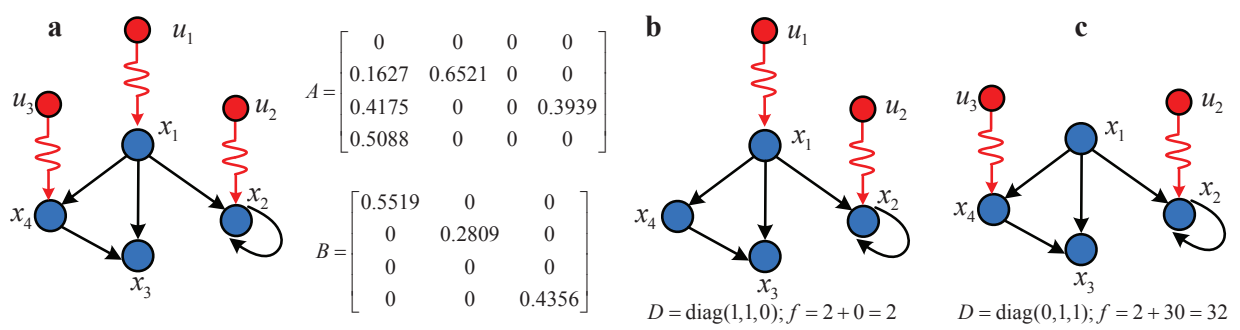

Figure 4: Fitness evaluation. (a) A directed-weighted network with 4 state nodes and 3 control nodes, the linking weights are randomized between 0 and 1 . (b) The fitness of control scheme $D=\operatorname{diag}(1,1,0)$ is 2 , of which the penalty is 0 , the network is fully controllable. (c) A slightly different control scheme from $(\mathbf{b}), D=\operatorname{diag}(0,1,1)$ with penalty $30 \neq 0$, the network is uncontrollable.

step 1. Initialize the global optimal solution $D_{\text {best }}=\operatorname{diag}\{1,1, \ldots, 1\}$ with $f_{\text {best }}\left(D_{\text {best }}\right)=P$. Randomly generate a population of $n$ solutions and set the number of generations $g=0$.

step 2. Evaluate the fitness $f\left(D_{i}\right)$ of each solution $D_{i}(i=1,2, \ldots, n)$ in the population. If $f\left(D_{i}\right)<$ $f\left(D_{\text {best }}\right)$, update $D_{\text {best }}=D_{i}$ and $f_{\text {best }}\left(D_{\text {best }}\right)=f\left(D_{i}\right)$.

step 3. Create a new population by repeating the following steps until its size reaches $n$ :

(a) select two parent solutions from current population according to their fitness (the smaller the fitness, the greater the chance to be selected);

(b) with a crossover probability $P_{c}$ cross over the parents to form two new children. If no crossover is performed, the two children are exact copies of their parents;

(c) with a mutation probability $P_{m}$ mutate the two new children at each gene;

(d) add the two children to the new population.

step 4. Replace the current population with the newly generated population.

step 5. If $g$ reaches the predefined maximum value, return $D_{\text {best }}$ and terminate; otherwise, set $g=g+1$, goto step 2 .

It is worth emphasizing that by the careful selection of $\sigma_{i}$ and algorithm initialization, we can make sure that the obtained best solution will always satisfy the PBH rank condition. First, with $\sigma_{i}=10 P$, it is easy to prove that $f(D) \leq P$ if and only if $D$ satisfies the controllability constraint (Eq.(12)). Second, with the initialization $f\left(D_{\text {best }}\right)=P$, whenever $D_{\text {best }}$ is updated by $D_{i}, f\left(D_{i}\right)<f\left(D_{\text {best }}\right)=P$, meaning that $D_{i}$ satisfies Eq.(12). In this way, the $D_{\text {best }}$ will always evolve along the path of feasible solutions that make the network fully controllable.

\subsection{Selection operator}

Selection operator is used to select parent chromosomes from a population for later crossover. In this paper, we adopt a hybrid selection strategy which combines the elitist selection and fitness proportionate selection. Specifically, we copy the top 5\% solutions directly to the next generation without crossover and mutation, then perform the conventional fitness proportionate selection. As the cost function is a minimization, we make a small modification, the selection probability $P_{i}$ of a chromosome $c_{i}$ is proportional to the inverse of its fitness $f_{i}$, i.e.

$$
P_{i}=\frac{1}{f_{i} \times S}, \quad i=1,2, \ldots, n
$$

where $f_{i}$ is the fitness of chromosome $c_{i}$ and $S=\sum_{i=1}^{n} 1 / f_{i}$. It is easy to prove that for $i \neq j$, $P_{i} f_{i}=P_{j} f_{j}=1 / S$, thus $P_{i} / P_{j}=f_{j} / f_{i}$. 


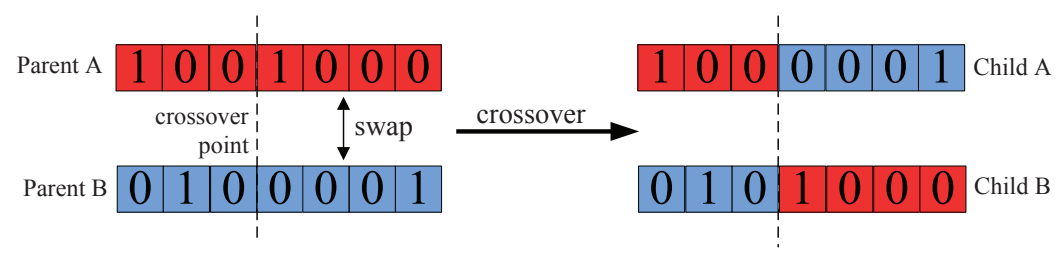

Figure 5: An example of single-point crossover operator of GA

\subsection{Crossover operator}

Crossover operator is used to produce offspring from parent chromosomes. Here we employ the single-point crossover, i.e., randomly choose a crossover point in $[1, p]$, where $p$ is the length of chromosome, swap the two fragments after crossover point to form two new children. Fig.5 shows an example of single-point crossover.

\subsection{Self-adaptive parameter $P_{c}$ and $P_{m}$}

As mentioned earlier, the traditional GA with fixed $P_{c}$ and $P_{m}$ is vulnerable to getting stuck at a local optimum. Therefore, we employ the adaptive GA [50], which utilizes the population information to adaptively adjust $P_{c}$ and $P_{m}$ so as to maintain the population diversity as well as to sustain the convergence capacity. $P_{c}$ and $P_{m}$ are increased when the population tends to get stuck at local optimum and are decreased when the population is scattered in the solution space.

To be specific, we detect the convergence with $\bar{f}-f_{\text {min }}$ to prevent the algorithm from converging to the local optimum, where $\bar{f}$ and $f_{\min }$ are the mean and best fitness of current population, respectively. When a population tends to get stuck at a local optimum, $\bar{f}-f_{\min }$ is likely to be less, thus $P_{c}$ and $P_{m}$ should be increased, i.e., $P_{c} \propto 1 /\left(\bar{f}-f_{\min }\right)$ and $P_{m} \propto 1 /\left(\bar{f}-f_{\min }\right)$. On the other hand, $P_{c}$ and $P_{m}$ should also vary with the fitness of individual solution. The solution with better fitness should have lower $P_{c}$ and $P_{m}$ whereas the solution with worse fitness should have higher $P_{c}$ and $P_{m}$, i.e., $P_{c} \propto\left(f-f_{\min }\right)$ and $P_{m} \propto\left(f-f_{\min }\right)$. Putting these two considerations together with the probability axiom, $P_{c}$ and $P_{m}$ are given as

$$
\begin{array}{ll}
P_{c}=\frac{k_{1}\left(f-f_{\text {min }}\right)}{\bar{f}-f_{\text {min }}}, & f \leq \bar{f} \\
P_{c}=k_{3}, & f>\bar{f}
\end{array}
$$

and

$$
\begin{array}{ll}
P_{m}=\frac{k_{2}\left(f-f_{\text {min }}\right)}{\bar{f}-f_{\text {min }}}, & f \leq \bar{f} \\
P_{m}=k_{4}, & f>\bar{f}
\end{array}
$$

where $k_{1}=k_{3}=1.0, k_{2}=k_{4}=0.5$, as suggested in [50].

\section{Results and discussions}

In this section, we demonstrate the performance and optimization results of the proposed GA and explore how network structure affects its controllability. In the following experiments, we 

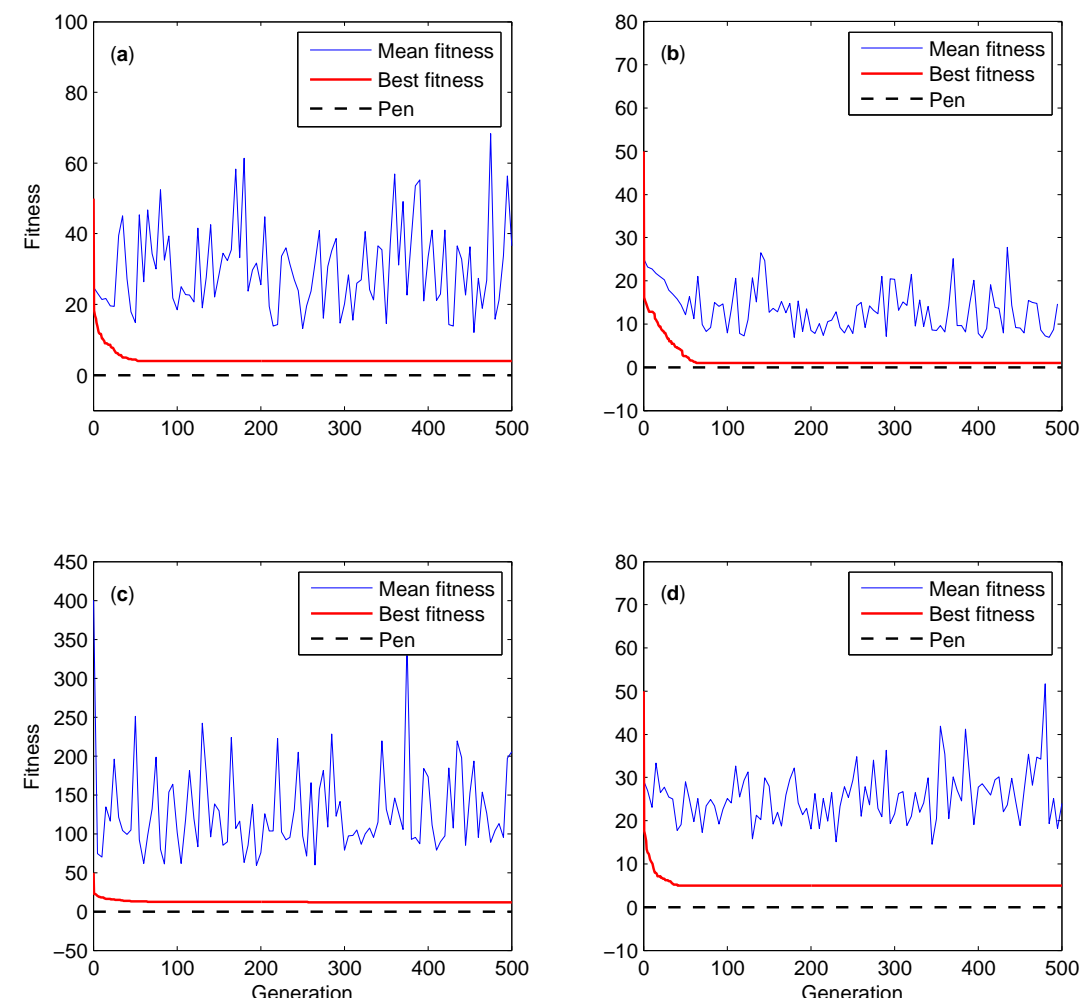

Figure 6: Plot of fitness as a function of generation for (a) ER with $\langle k\rangle=3$, (b) ER with $\langle k\rangle=5$, (c) SF with $\langle k\rangle=3$ and $\gamma=2.2$, and (d) SF with $\langle k\rangle=5$ and $\gamma=2.2$. The blue line represents the mean fitness of current generation, the red line denotes the fitness of $D_{\text {best }}$ and the black dashed line denotes the corresponding penalty of $D_{\text {best }}$. The networks are directed with $N=P=50$ and the results are averaged over 10 independent realizations.

use the canonical Erdős-Rényi [52] (ER) random and scale-free [12] (SF) networks as benchmarks. The networks are generated from the static model [53] with $N$ state nodes and $P$ control nodes $(P=N)$, each control node is linked to state nodes with uniform probability and the weights of all links are randomized between 0 and 1 . The number of selected control nodes is denoted as $N_{c}$ and the density of selected control nodes is denoted as $n_{c}$ where $n_{c}=N_{c} / N$. The minimum value of $N_{c}$ and $n_{c}$ is denoted as $N_{c m}$ and $n_{c m}$, respectively.

\subsection{Performance of the proposed GA}

First, in order to validate our previous claim that the obtained best solution will always satisfy the PBH rank condition, i.e., $\operatorname{Pen}\left(D_{\text {best }}\right)=0$. We test the algorithm on different networks, the results are shown in Fig.6. It can be seen that although the mean fitness fluctuates dramatically, which can be attributed to the self-adaptive parameters strategy $\left(P_{c}\right.$ and $\left.P_{m}\right)$, the fitness of obtained best solution quickly converges to the minimum value after about 50 generations. 
More importantly, the penalty $\operatorname{Pen}\left(D_{\text {best }}\right)$ always equals zero, meaning that the obtained solution always satisfies the PBH rank condition and therefore can provide full control over the network.

Table 1: Performance comparison between the proposed GA and EO [38]. For each network, we show the number of state and control nodes $(N / P)$ and average degree $(\langle k\rangle)$. For each algorithm, we show the minimum number of control nodes it achieves $\left(N_{c m}\right)$, the number of iterations it takes (Iterations) and the time it consumes (Time). The SF networks have $\gamma=2.2$ and the results are averaged over 10 independent realizations.

\begin{tabular}{cccccccccc}
\hline \hline & & \multicolumn{3}{c}{ The proposed GA } & \multicolumn{3}{c}{ EO [38] } & Time \\
\cline { 5 - 8 } Network & $N / P$ & $\langle k\rangle$ & $N_{c m}$ & Iterations & Time & $N_{c m}$ & Iterations & Time & saved \\
\hline ER & 25 & 1.5 & 2 & 30 & $0.23 \mathrm{~s}$ & 3 & 22 & $0.45 \mathrm{~s}$ & $48.78 \%$ \\
ER & 50 & 3.0 & 3 & 34 & $4.19 \mathrm{~s}$ & 3 & 22 & $7.79 \mathrm{~s}$ & $46.28 \%$ \\
ER & 75 & 3.5 & 5 & 55 & $19.63 \mathrm{~s}$ & 5 & 23 & $43.67 \mathrm{~s}$ & $55.04 \%$ \\
ER & 100 & 4.0 & 5 & 75 & $66.26 \mathrm{~s}$ & 6 & 24 & $121.62 \mathrm{~s}$ & $45.52 \%$ \\
ER & 125 & 4.5 & 6 & 90 & $179.84 \mathrm{~s}$ & 6 & 26 & $312.66 \mathrm{~s}$ & $42.48 \%$ \\
ER & 150 & 5.0 & 8 & 101 & $398.03 \mathrm{~s}$ & 10 & 36 & $728.04 \mathrm{~s}$ & $45.32 \%$ \\
SF & 25 & 1.5 & 3 & 20 & $0.43 \mathrm{~s}$ & 4 & 22 & $0.75 \mathrm{~s}$ & $43.07 \%$ \\
SF & 50 & 3.0 & 5 & 32 & $4.83 \mathrm{~s}$ & 5 & 21 & $8.37 \mathrm{~s}$ & $42.26 \%$ \\
SF & 75 & 3.5 & 6 & 50 & $18.80 \mathrm{~s}$ & 6 & 22 & $36.85 \mathrm{~s}$ & $48.98 \%$ \\
SF & 100 & 4.0 & 9 & 70 & $73.93 \mathrm{~s}$ & 10 & 21 & $117.58 \mathrm{~s}$ & $37.12 \%$ \\
SF & 125 & 4.5 & 18 & 85 & $187.56 \mathrm{~s}$ & 20 & 26 & $317.24 \mathrm{~s}$ & $40.88 \%$ \\
SF & 150 & 5.0 & 37 & 113 & $406.76 \mathrm{~s}$ & 40 & 30 & $709.40 \mathrm{~s}$ & $42.67 \%$ \\
\hline \hline
\end{tabular}

Next, we compare the performance of our proposed GA with that of the extreme optimization (EO) algorithm proposed in [38], the results are displayed in Table 1. Comparing the two columns of $N_{c m}$, we can see that both algorithms converge to almost the same $N_{c m}$, indicating their good performance of finding the minimal control nodes. Nevertheless, our algorithm outperforms EO a little under some cases, e.g., ER with $N=100,150$ and SF with $N=125,150$. As for time efficiency, it can be seen that although the convergence speed (number of iterations) of EO is faster than our algorithm, our algorithm saves about $40 \%$ time than EO, which may be confusing at the first glance. The reason is that, the most time-consuming step for both algorithms is controllability test, however, the rank computation of Kalman matrix $(N \times N P)$ costs much more time than that of PBH matrix $(N \times(N+P))$, also the formation of Kalman matrix contains massive matrix multiplications that are computationally expensive. As a result, EO costs much more time than GA in one step of iteration. In total, the single-step time saving of GA defeats its increase of iterations, leading to the result that the proposed GA is more efficient than EO.

Finally, as GA is a heuristic algorithm that provides approximate solutions and will not guarantee to find the optimal solution, it is necessary to examine the accuracy of its solution. Take into account that the optimal solution is unknown, we use the solution calculated by the structural controllability theory [23] as the benchmark. We compare the minimum number of control nodes obtained by GA with the optimum under different networks, the results are shown in Fig.7. It can be seen that for both ER and SF networks, the $N_{c m}$ obtained by GA always equals to the optimum, indicating that our proposed approach actually finds the optimal solution despite its nature of approximation, which is an ideal result beyond our expectations.

\subsection{Results of the proposed GA}

In order to show the optimization results and demonstrate the evolution process of network topology, we apply GA to directed ER and SF networks, the results are shown in Fig.8. From 

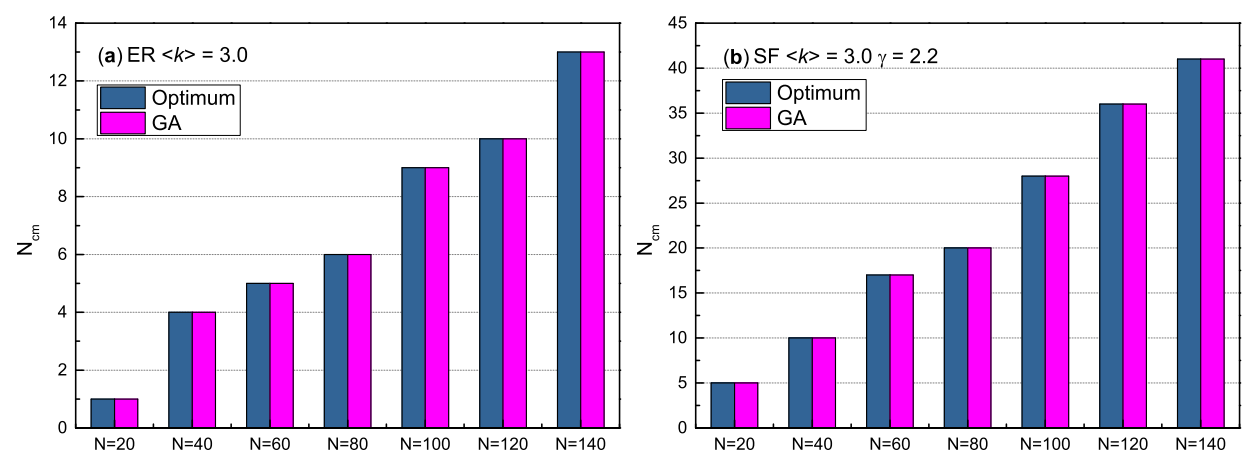

Figure 7: Comparisons of the minimum number of control nodes obtained by GA with the optimum for (a) directed ER networks with $\langle k\rangle=3.0$, (b) directed SF networks with $\langle k\rangle=3.0$ and $\gamma=2.2$. The optimum values are calculated by the structural controllability theory [23] and the results are averaged over 10 independent realizations.

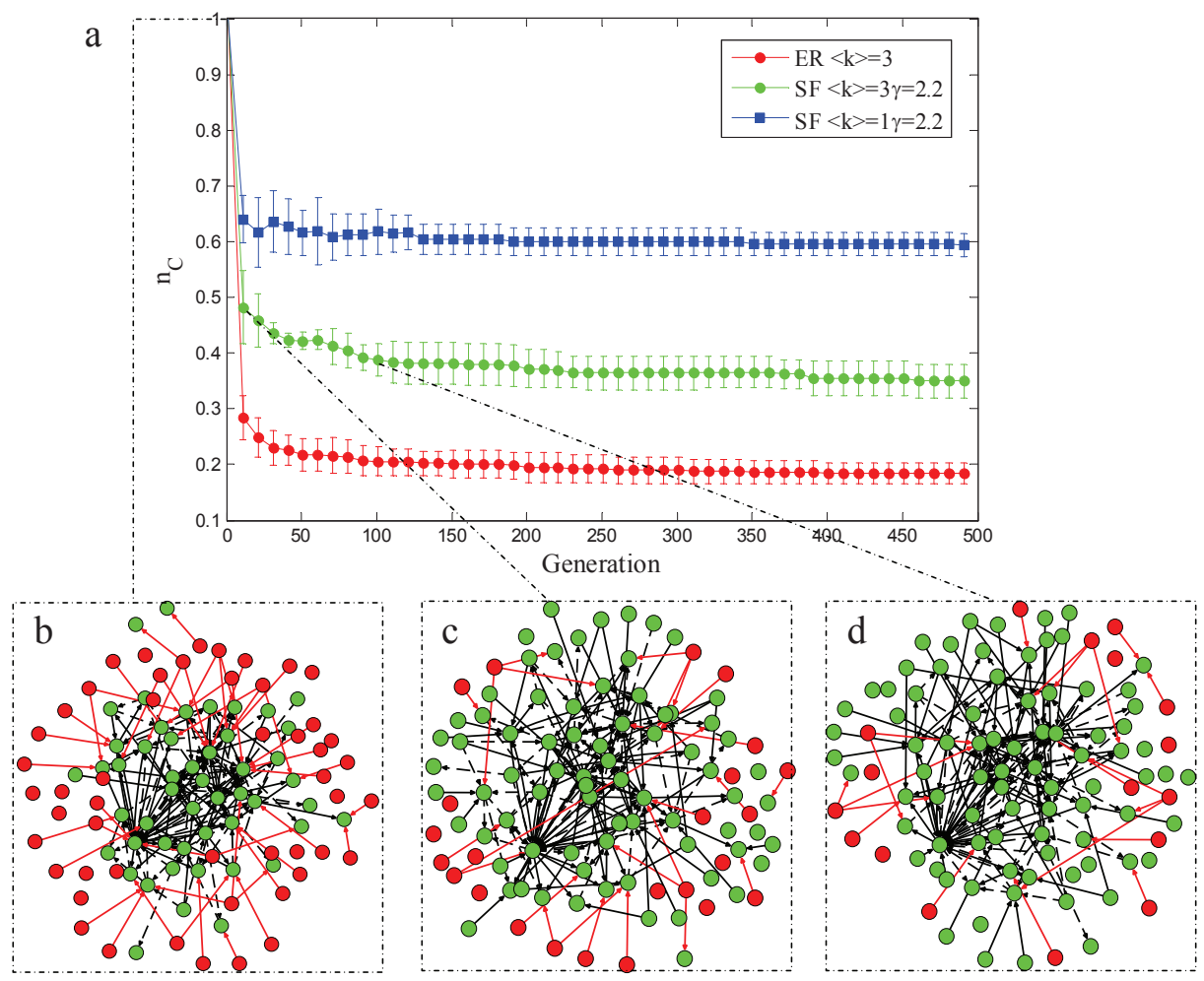

Figure 8: GA optimization process on directed ER and SF networks and the evolution process of network topology. (a), The convergence process of $n_{c}$ on ER and SF networks with $N=P=50$. The results are averaged over 10 independent runs, the error bars denote the standard deviation. (b), The network topology at the 0th generation (initial topology) on SF case with $\gamma=2.2$ and $\langle k\rangle=3$. The selected control nodes are marked with red and the other nodes are marked with green. Red links are from control nodes to state nodes and black links are between state nodes. (c), The network topology at the 11th generation. (d), The network topology at the 101th generation. 

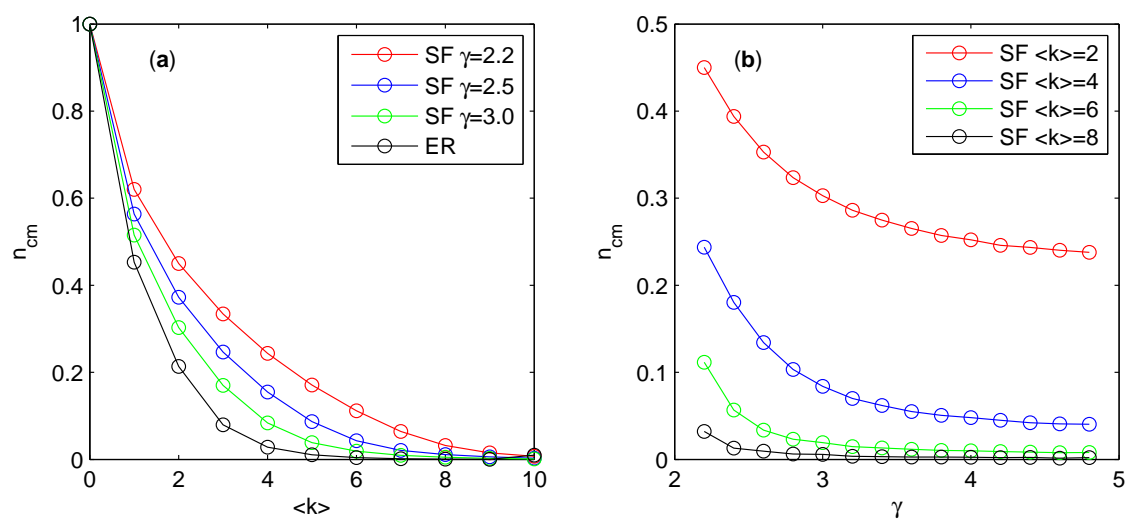

Figure 9: The structural impact on the minimum number of control nodes for directed networks. (a) $n_{c m}$ as a function of $\langle k\rangle$ for ER and SF networks with fixed $\gamma$. (b) $n_{c m}$ as a function of $\gamma$ for SF networks with fixed $\langle k\rangle$. The ER and SF networks have $N=P=50$ and the results are averaged over 100 independent realizations.

Fig.8a we can see that on every case, $n_{c}$ decreases sharply at the first few generations, then quickly converges to its minimum value $n_{c m}$, revealing the effectiveness of the proposed scheme. Taking the ER case with $\langle k\rangle=3$ as an example, $n_{c}$ quickly converges to 0.2 after about 50 generations, indicating that 10 control nodes are enough to maintain the full controllability of the network. In contrast, for the SF case with $\gamma=2.2$ and $\langle k\rangle=3, n_{c}$ converges to 0.4 after about 130 generations, meaning that one has to control about 20 nodes to control the whole network. Fig.8b-d together capture this case's topology evolution at the 0th, 11th, and 101th generation, from which the declining trend of control nodes can be grasped more intuitively. Notice that the two SF cases with same $\gamma$ but different $\langle k\rangle$ converge to different $n_{c m}$, whereas the ER and SF cases with same $\langle k\rangle$ also converge to different $n_{c m}$. This prompts us that the minimum number of control nodes $N_{c m}$ may be affected by average degree $\langle k\rangle$ and power-law exponent $\gamma$.

To confirm our conjecture, we plot $n_{c m}$ as a function of $\langle k\rangle$ and $\gamma$ in Fig.9. From Fig.9a, we can see that for fixed $\gamma, n_{c m}$ decreases with $\langle k\rangle$ monotonically and more and more slowly until $\langle k\rangle$ exceeds a relatively large value, after that $n_{c m}$ goes flat. This asymptotic exponential dependence of $n_{c m}$ on $\langle k\rangle$ indicates that the denser a network is, the fewer control nodes are needed to control it. From Fig.9b, it can be seen that for fixed $\langle k\rangle, n_{c m}$ decreases as $\gamma$ increases, suggesting that $n_{c m}$ may be affected by degree heterogeneity, which measures the spread of degrees between the less connected nodes and the more connected nodes. Here we define the degree heterogeneity as $H=\left[\sum_{i}\left(k_{i}-\langle k\rangle\right)^{2} / N\right]^{1 / 2}(i=1,2, \ldots, N)$, where $k_{i}$ denotes the degree of node- $i$. It is obvious that the degree heterogeneity is zero $(H=0)$ for random regular networks, in which all nodes have the same degree. For SF networks, the larger $H$ corresponds to smaller $\gamma$ due to the long tail of degree distribution. In Fig. 10, we plot $n_{c m}$ as a function of $H$, it is clear that $n_{c m}$ increases monotonically with $H$, indicating that the more heterogeneous a network is, the more control nodes are needed to control the network, which agrees with the conclusion in [38].

Putting these results together, we find that given a network consisting of both state nodes and control nodes, the denser the network is, the fewer control nodes are needed to control it. Furthermore, the larger differences between node degrees, the more control nodes are needed to control the network. In short, networks that are sparse and heterogeneous are the most difficult to control. It should be stressed that although we obtain this conclusion by applying GA on directed 


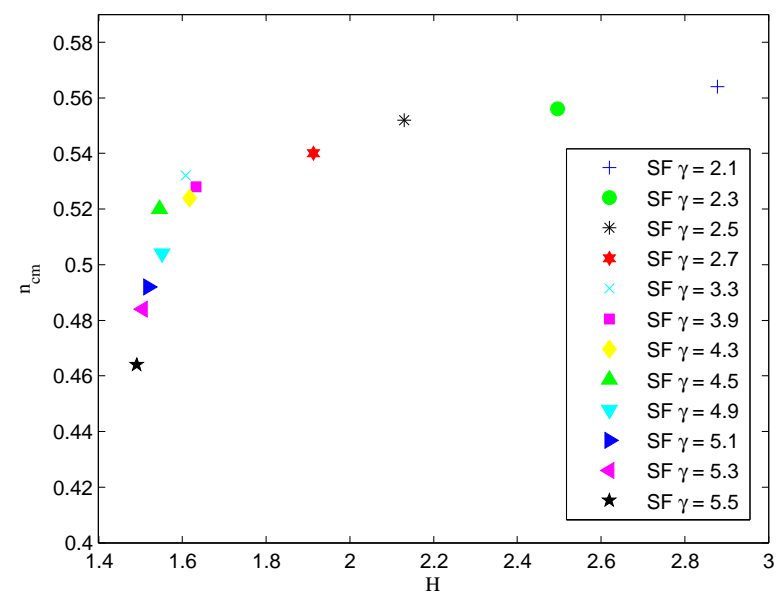

Figure 10: The impact of degree heterogeneity on the minimum number of control nodes. $n_{c m}$ as a function of $H$ for SF networks with different $\gamma$.

networks, we have verified that the conclusion is also valid for undirected networks.

\section{Conclusions}

Controlling complex networks is one of the major challenges in the field of network science. In this paper, we develop a novel GA oriented heuristic tool to address the controllability of arbitrary networks consisting of both state nodes and control nodes under PBH rank condition. The proposed method can be applicable to arbitrary networks without any limitations and is more efficient than the previous best heuristic algorithm [38]. Experimental results on popular model networks show the effectiveness of this method and the evolution process of network topology is captured. In addition, we explore how network structure affects its controllability and find that the minimum number of control nodes is mainly affected by the network's average degree and degree heterogeneity. The sparser a network is, the more control nodes are needed to control it; the more heterogeneous a network is, the more control nodes are needed to control the network. Briefly, networks that are sparse and heterogeneous are the most difficult to control. In order to simplify the problem, we have intentionally ignored the robustness issue, the future work will aim to design the robust network topology with the minimal control nodes while maintaining its full controllability.

\section{Acknowledgments}

This work was supported by the National Science Foundation of China under grant No. 61171150.

\section{References}

[1] S. H. Strogatz, Exploring complex networks, Nature 410 (6825) (2001) 268-276. 
[2] R. Albert, A.-L. Barabási, Statistical mechanics of complex networks, Reviews of modern physics 74 (1) (2002) 47

[3] S. N. Dorogovtsev, A. V. Goltsev, J. F. F. Mendes, Pseudofractal scale-free web, Physical Review E 65 (6) (2002) 066122.

[4] M. E. Newman, The structure and function of complex networks, SIAM review 45 (2) (2003) 167-256.

[5] J. A. Dunne, R. J. Williams, N. D. Martinez, Food-web structure and network theory: the role of connectance and size, Proceedings of the National Academy of Sciences 99 (20) (2002) 12917-12922.

[6] S. A. Kauffman, Metabolic stability and epigenesis in randomly connected nets.

[7] T. Ito, T. Chiba, R. Ozawa, M. Yoshida, M. Hattori, Y. Sakaki, A comprehensive two-hybrid analysis to explore the yeast protein interactome, Proceedings of the National Academy of Sciences 98 (8) (2001) 4569-4574.

[8] D. Knoke, S. Yang, Social network analysis, Vol. 154, Sage, 2008.

[9] D. J. De Solla Price, Is technology historically independent of science? a study in statistical historiography, Technology and Culture (1965) 553-568.

[10] S. Redner, How popular is your paper? an empirical study of the citation distribution, The European Physical Journal B-Condensed Matter and Complex Systems 4 (2) (1998) 131-134.

[11] D. J. Watts, S. H. Strogatz, Collective dynamics of small-worldnetworks, nature 393 (6684) (1998) $440-442$.

[12] A.-L. Barabási, R. Albert, Emergence of scaling in random networks, science 286 (5439) (1999) 509-512.

[13] A. Arenas, A. Díaz-Guilera, J. Kurths, Y. Moreno, C. Zhou, Synchronization in complex networks, Physics Reports 469 (3) (2008) 93-153

[14] S. Boccaletti, V. Latora, Y. Moreno, M. Chavez, D.-U. Hwang, Complex networks: Structure and dynamics, Physics reports 424 (4) (2006) 175-308

[15] G. Palla, I. Derényi, I. Farkas, T. Vicsek, Uncovering the overlapping community structure of complex networks in nature and society, Nature 435 (7043) (2005) 814-818.

[16] J. Duch, A. Arenas, Community detection in complex networks using extremal optimization, Physical review E 72 (2) (2005) 027104.

[17] A. Lancichinetti, S. Fortunato, J. Kertész, Detecting the overlapping and hierarchical community structure in complex networks, New Journal of Physics 11 (3) (2009) 033015.

[18] R. Pastor-Satorras, A. Vespignani, Epidemic dynamics and endemic states in complex networks, Physical Review E 63 (6) (2001) 066117.

[19] M. Boguná, R. Pastor-Satorras, Epidemic spreading in correlated complex networks, Physical Review E 66 (4) (2002) 047104

[20] A. Barrat, M. Barthelemy, A. Vespignani, Dynamical processes on complex networks, Vol. 1, Cambridge University Press Cambridge, 2008.

[21] R. Albert, H. Jeong, A.-L. Barabási, Error and attack tolerance of complex networks, Nature 406 (6794) (2000) $378-382$

[22] R. Cohen, S. Havlin, Complex networks: structure, robustness and function, Cambridge University Press, 2010.

[23] Y.-Y. Liu, J.-J. Slotine, A.-L. Barabási, Controllability of complex networks, Nature 473 (7346) (2011) $167-173$.

[24] X. F. Wang, G. Chen, Pinning control of scale-free dynamical networks, Physica A: Statistical Mechanics and its Applications 310 (3) (2002) 521-531.

[25] F. Sorrentino, M. di Bernardo, F. Garofalo, G. Chen, Controllability of complex networks via pinning, Physical Review E 75 (4) (2007) 046103.

[26] A. Lombardi, M. Hörnquist, Controllability analysis of networks, Physical Review E 75 (5) (2007) 056110

[27] B. Liu, T. Chu, L. Wang, G. Xie, Controllability of a leader-follower dynamic network with switching topology, Automatic Control, IEEE Transactions on 53 (4) (2008) 1009-1013.

[28] A. Rahmani, M. Ji, M. Mesbahi, M. Egerstedt, Controllability of multi-agent systems from a graph-theoretic perspective, SIAM Journal on Control and Optimization 48 (1) (2009) 162-186.

[29] D.-H. Kim, A. E. Motter, Slave nodes and the controllability of metabolic networks, New Journal of Physics 11 (11) (2009) 113047

[30] T. Nepusz, T. Vicsek, Controlling edge dynamics in complex networks, Nature Physics 8 (7) (2012) $568-573$.

[31] G. Yan, J. Ren, Y.-C. Lai, C.-H. Lai, B. Li, Controlling complex networks: How much energy is needed?, Physical Review Letters 108 (21) (2012) 218703

[32] Y.-Y. Liu, J.-J. Slotine, A.-L. Barabási, Control centrality and hierarchical structure in complex networks, Plos one 7 (9) (2012) e44459.

[33] C.-L. Pu, W.-J. Pei, A. Michaelson, Robustness analysis of network controllability, Physica A: Statistical Mechanics and its Applications 391 (18) (2012) 4420-4425.

[34] W.-X. Wang, X. Ni, Y.-C. Lai, C. Grebogi, Optimizing controllability of complex networks by minimum structural perturbations, Physical Review E 85 (2) (2012) 026115

[35] H. Lv-Lin, L. Song-Yang, L. Gang, B. Liang, Controllability and directionality in complex networks, Chinese Physics Letters 29 (10) (2012) 108901 
[36] Y.-D. Xiao, S.-Y. Lao, L.-L. Hou, L. Bai, Edge orientation for optimizing controllability of complex networks, Physical Review E 90 (4) (2014) 042804.

[37] F. L. Iudice F. Garofalo, F. Sorrentino, Structural nermeability of complex networks to control sionals Nature

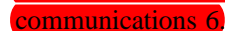

[38] J. Ding, Y.-Z. Lu, J. Chu, Studies on controllability of directed networks with extremal optimization, Physica A: Statistical Mechanics and its Applications 392 (24) (2013) 6603-6615.

[39] R. E. Kalman, Mathematical description of linear dynamical systems, Journal of the Society for Industrial \& Applied Mathematics, Series A: Control 1 (2) (1963) 152-192.

[40] Z. Yuan, C. Zhao, Z. Di, W.-X. Wang, Y.-C. Lai, Exact controllability of complex networks, Nature communications

[41] M. Hautus, Stabilization controllability and observability of linear autonomous systems, in: Indagationes Mathematicae (Proceedings), Vol. 73, Elsevier, 1970, pp. 448-455.

[42] C.-T. Lin, Structural controllability, IEEE Transactions on Automatic Control 19 (1974) 201-208. doi:10.1109/TAC.1974.1100557.

[43] R. W. Shields, J. B. Pearson, Structural controliability of multi-input linear systems, in: Conference on Decision and Control, 1975. doi:10.1109/CDC.1975.270615.

[44] J. E. Hopcroft, R. M. Karp, An n5/2 Algorithm for Maximum Matchings in Bipartite Graphs, Siam Journal on Computing 2 (1973) 225-231.

[45] E. D. Sontag, Mathematical control theory: deterministic finite dimensional systems, Vol. 6, Springer, 1998.

[46] M. Hautus, Controllability and observability conditions of linear autonomous systems, PROCEEDINGS OF THE KONINKLIJKE NEDERLANDSE AKADEMIE VAN WETENSCHAPPEN SERIES A-MATHEMATICAL SCIENCES 72 (5) (1969) 443.

[47] W. J. Rugh, Linear system theory, Prentice-Hall, Inc., 1996.

[48] P. J. Antsaklis, A. N. Michel, Linear systems, Springer, 2006.

[49] J. H. Holland, J. S. Reitman, Cognitive systems based on adaptive algorithms, ACM SIGART Bulletin (63) (1977) 49-49.

[50] M. Srinivas, L. M. Patnaik, Adaptive probabilities of crossover and mutation in genetic algorithms, Systems, Man and Cybernetics, IEEE Transactions on 24 (4) (1994) 656-667.

[51] P. Nanakorn, K. Meesomklin, An adaptive penalty function in genetic algorithms for structural design optimization, Computers \& Structures 79 (29) (2001) 2527-2539.

[52] P. Erdôs, A. Rényi, On the strength of connectedness of a random graph, Acta Mathematica Hungarica 12 (1) (1961) 261-267.

[53] K.-I. Goh, B. Kahng, D. Kim, Universal behavior of load distribution in scale-free networks, Physical Review Letters 87 (27) (2001) 278701 